\title{
La part du père dans les interruptions spontanées répétées de grossesse (isrg) d'origine immunologique
}

\author{
M. REZNIKOFF-ETIEVANT \\ Sces de Gynécologie Obstétrique et d'Embryo-Pathologie, \\ Hôpital St Antoine - 184 rue du Fg St Antoine 75012 Paris
}

La part du père est bien évidemment très importante en ce qui concerne les causes immunologiques des ISRG. Les phénomènes immunologiques sont en grande partie le résultat d'une stimulation par des antigènes étrangers: c'est l'alloimmunisation, ou bien ils sont responsables d'un dysfonctionnement du système immunitaire: c'est l'autoimmunité.

$\mathrm{Au}$ cours de la grossesse les phénomènes alloimmuns sont importants puisque l'organisme maternel doit reconnaître les alloantigènes paternels $d u$ fotus pour établir un état d'immunotolérance vis à vis du foetus. Ce mécanisme est bien connu depuis 1976 avec Beer et Billingham qui, cherchant à provoquer des résorptions embryonnaires chez la souris, ont injecté des cellules, provenant de la souris mâle, aux femelles pour les alloimmuniser avant de les croiser avec ces mâles. Contrairement à toute attente, le phénomène inverse se produisit : non seulement ils n'obtinrent pas d'avortement mais les placentas se développèrent de façon significativement plus importante. L'alloimmunisation paraît donc favoriser l'évolution de la gestation.

En fait, pendant la grossesse le système immunitaire est modifié et l'on assiste d'une part à une diminution de l'immunité cellulaire avec amélioration de certaines maladies à médiation cellulaire comme l'arthrite rhumatoïde et d'autre part à l'accen- tuation de l'immunité à médiation anticorps avec aggravation de certaines maladies à médiation anticorps comme le lupus érythémateux disséminé.

Certains antigènes contrôlés par des gènes provenant du père pourraient jouer un rôle probablement important dans l'installation de la tolérance de la mère au foetus. Par ailleurs, ces alloantigènes peuvent également, comme dans toute alloimmunisation, stimuler la production d'autoanticorps chez les femmes particulièrement susceptibles d'en développer.

Les interruptions spontanées de grossesse sont banales lorsqu'elles sont isolées chez une même patiente. Le phénomène devient très préoccupant lorsqu'il devient récurrent. Environ 1 couple sur 100 présente des ISRG (au moins 3 ISG consécutives). Il est actuellement admis que dans environ la moitié des cas un phénomène immunologique est à l'origine de ces échecs de grossesse.

Plusieurs causes immunologiques sont décrites depuis déjà une décennie. Elles sont de deux ordres:

1) les causes alloimmunes qui relèvent, pour la presque totalité des cas, d'une insuffisance de stimulation des mécanismes de tolérance.

2) les causes autoimmunes qui se produisent sous l'effet stimulant de l'embryon allogénique. 


\section{LES CAUSES ALLOIMMUNES.}

Le rôle du père est évident en ce qui concerne une éventualité rarissime: l'alloimmunisation dans le système $P$ (connu par ses antigènes érythrocytaires). Les antigènes de ce groupe sont présents sur le trophoblaste qui est directement en contact avec le sang maternel et la muqueuse déciduale. Il existe de très rares femmes de phénotype pp qui n'expriment aucun des antigènes du système $\mathrm{P}$ et produisent donc les anticorps correspondants. Le fœetus portant les différents antigènes $\mathrm{P}$ du père sera rejeté par les anticorps maternels. La prévention de ces accidents de la grossesse, par épuration des anticorps maternels circulants, soit par plasmaphérèse, soit par absorption des anticorps sur des hématies $\mathrm{P}$, a été suivi de grossesses normalement évolutives [11]. Notons que l'anticorps anti-P1 très fréquent chez les sujet de sous groupe P2, n'a jamais été incriminé dans les ISRG.

En fait, les causes alloimmunes sont dues essentiellement à un manque de production des facteurs immunologiques de la tolérance maternelle pour son fœtus. L'hypothèse d'un tel mécanisme repose sur les observations suivantes:

\section{Dans l'espèce humaine, il est fré- quent d'observer}

1) qu'une femme peut faire des ISRG avec un conjoint et pas avec un autre;

2) dans de très grandes cohortes, il a été possible d'observer une plus forte compatibilité HLA (Histocompatibility Leucocyte Antigen) ente les conjoints de couples avec ISRG par rapport aux couples ayant plusieurs enfants et pas d'ISG [9];

3) une absence ou une diminution des anticorps bloquants, d'antiidiotypes, d'anticorps facilitants et d'antipaternels (AAP) dirigés contre les lymphocytes du conjoint;

4) la facilitation des greffes intra-familiales par transfusion, préalable à la greffe, du sang du donneur d'organe.

\section{Chez les animaux :}

1) la stimulation allogénique par des cellules de la souris mâle avant leur croisement avec les souris femelles, favorise la croissance placentaire;

2) certaines souches de souris présentent de nombreuses résorptions embryonnaires lorsqu'elles sont croisées avec certaines souches de mâles; si on les immunise préalablement avec des cellules du mâle ou d'une autre souche mais antigéniquement voisine, les résorptions embryonnaires sont fortement et significativement diminuées;

3) en situation inter-espèces alors que la gestation est vouée à l'échec, il a été possible d'obtenir des naissances par stimulation allogénique avec des cellules provenant du mâle.

Ces observations ont conduit certains chercheurs dont nous même en France, à prévenir une nouvelle récidive d'ISG, chez les femmes avec ISRG, en leur injectant, préalablement à une nouvelle grossesse, les lymphocytes de leur conjoint. Une métaanalyse internationale a été effectuée à patir des essais réalisés en double insu par différentes équipes $[1,10]$. Seules les femmes ayant présenté au moins 3 ISG consécutives avec le même partenaire et sans enfant vivant ont été retenues, ces ISG n'ayant aucune cause classique de fausse couche spontanée et les femmes dépourvues d'autoanticorps antiphospholipide et d'anticorps antipaternel . Avant une nouvelle grossesse dans un premier groupe, les femmes ont reçu une seule injection de lymphocytes de leur conjoint tandis que dans le groupe placebo elles ont reçu une injection de leurs propres lymphocytes. Cette étude a permis de montrer que le traitement

- a une efficacité significative, mais faible puisqu'il faut traiter 7 patientes pour espérer être efficace dans un cas supplémentaire. 
- cette efficacité est moindre si des autoanticorps apparaissent.

- les complications du traitement ne diffèrent pas de façon importante entre les deux groupes. Les effets à long terme ne sont pas évalués.

Ces résultats indiquent que:

1) la sélection des patientes à traiter est insuffisante.

2) les modalités thérapeutiques ne sont pas optimales.

3) le dépistage d'autres anomalies, par exemple autoimmunes, n'apparaissant qu'en début de grossesse ou après injection de lymphocytes, est insuffisant.

Concernant les modalités thérapeutiques, nous avions montré que si l'on répétait les injections de lymphocytes du mari 2 ou 3 fois, pour favoriser une séroconversion des AAP non apparues après la première injection, nous pouvions augmenter le taux des réussites, celui-ci passant de $64,3 \%$ à $77 \%$ [8].

Concernant une meilleure sélection des patientes, il est certain qu'elle s'impose, tant sur le plan clinique que biologique. Probablement d'autres facteurs non encore identifiés sont à démontrer; ces recherches font l'objet d'études dans notre laboratoire. Une connaissance plus approfondie des ISG associées à des anomalies autoimmunes peut apporter sur ce plan également un important bénéfice.

\section{LES CAUSES AUTOIMMUNES}

L'ensemble de l'unité fœtoplacentaire portant des antigènes étrangers provenant $d u$ père peut favoriser, comme toute greffe ou transfusion allogénique, la survenue d'autoanticorps ou renforcer ceux qui préexistent à la grossesse. De surcroît la grossesse favorise la réponse immunitaire à anticorps.
L'on sait depuis de nombreuses années qu'au cours du LED en phase aiguë de la maladie, le taux de ISG est 3 fois plus élevé et que la grossesse est un facteur d'aggravation de la maladie. Mais l'accident gravidique peut survenir en dehors de maladie autoimmune avérée et déjà en 1975 Nilson met en évidence la relation entre mort fœetale in utero et présence d'anticoagulants circulants (ACC).

Par la suite, Soulier et Boffa ont décrit un syndrome associant la présence d'ACC, de thromboses et d'avortements répétés. Enfin, plus récemment un certain nombre de phénomènes pathologiques ont été rassemblés sous le nom de "syndrome des antiphospholipides" (SaPL). Celui-ci est défini par la coexistence d'un facteur biologique (présence d'anticorps anticardiolipines et/ou ACC) et d'un facteur clinique (thrombose veineuse et/ou artérielle et/ou avortements spontanés répétés).

Depuis quelques années, on observe un regain d'intérêt pour les antiphospholipides étant donné les progrès effectués dans leur dépistage et la variété de leurs implications cliniques : thromboses veineuses ou artérielles, cardiaques, cérébrales, pulmonaires etc. Enfin l'expérimentation animale récente permet de mieux comprendre les mécanismes d'action de ces antiphospholipides dans la genèse d'ISG et même d'entrevoir leur possible action néfaste à un stade très précoce du développement embryonnaire.

\section{Les antiphospholipides (aPL)}

Les aPL sont une famille d'anticorps divers dont le point commun est de se fixer sur certains phospholipides et donc sur de nombreux tissus et en ce qui intéresse la grossesse: les plaquettes, l'endothélium vasculaire, les facteurs de la coagulation, mais aussi le trophoblaste.

Les anticorps antiphospholipides doivent être systématiquement recherchés dans les accidents gravidiques à répétition en particulier s'il n'existe aucune cause connue. 
Leur identification se fait par la recherche d'un ACC (les tests de thromboplastine activée et diluée avec tests de neutralisation), d'anticardiolipines IgG, IgM IgA et d'anti-32GP1, de facteurs antinucléaires sur cellules humaines ( $\mathrm{HeP} 2$,) d'anti-DNA ainsi que les tests TPHA et VDRL. La recherche d'autres autoanticorps peut aussi avoir un interêt, en particulier les anticorps anti-plaquettes en cas de thrombopénie, les anti-thyroïdiens et les facteurs rhumatoïdes.

Dans l'étude d'une série de 252 femmes qui présentaient des ISRG, nous avons pu montrer chez $46 \%$ d'entre elles, en dehors de toute grossesse ou en tout début, la présence d'un ou plusieurs antiphospholipides contre moins de $8 \%$ dans une population de femmes enceintes sans antécédents d'ISG.

Chez les femmes avec ISRG, il convient de contrôler en tout début de la grossesse ce bilan; en effet nous avons pu observer que $28 \%$ des femmes qui ne possèdent pas d'antiphospholipides en dehors de la grossesse, pouvaient développer un ou plusieurs de ces anticorps en tout début de celle-ci.

\section{Quels sont leurs mécanismes d'action?}

\section{En expérimentation animale :}

- Le transfert passif d'anticorps anticardiolipines monoclonaux ou humains induit chez la souris un véritable syndrome des antiphospholipides (SaPL) avec résorptions embryonnaires, antiphospholipides sériques persistants et thrombopénies.

- Fait intéressant, l'injection de gammaglobulines polyvalentes humaines chez la souris présentant un SaPL induit, prévient la survenue des résorptions embryonnaires.

\section{Les aPL favorisent la survenue de thromboses}

- Contrairement à ce que leur nom pourrait laisser entendre, les anticoagulants circulants et autres aPL favorisent la survenue de thromboses vasculaires diverses, notamment placentaires et déciduales chez la femme enceinte. Notons que la grossesse favorise leur apparition et amplifie ceux qui préexistent.

- La survenue de thromboses s'expliquerait par une défaillance du système fibrinolytique, l'inhibition de la formation de prostacycline et une interférence entre aPL et le système thrombomoduline et protéine $\mathrm{C}$. En fait, la survenue de thromboses vasculaires n'expliquerait que des accidents relativement tardifs de la grossesse de la fin du premier trimestre et surtout 2ème et 3ème trimestres, ISG tardives, morts fœtales in utero, retard de croissance in utero, prééclampsie.

\section{Traitements préventifs des ISRG avec autoanticorps}

Parmi les traitements proposés dans la prévention des accidents de la grossesse associés aux antiphospholipides (corticoïdes, aspirine, héparine et gammaglobulines polyvalentes IV), il est bien difficile de dire actuellement lequel d'entre eux est le plus efficace [8]. Les études à ce sujet varient énormément quant à la sélection clinique des patientes et les tests biologiques utilisés.

- Les corticoïdes se sont avérés efficaces pour la majorité des études, où ils ont été le plus souvent associés à de faibles doses d'aspirine. Les corticoïdes agissent sur la synthèse des anticorps, mais leurs inconvénients sont leurs effets secondaires

- l'aspirine à faible dose, prévient la formation de thromboses responsables d'ISG tardives, de morts fœtales in utero, mais ne peut théoriquement pas agir sur les accidents non thrombotiques éventuels des ISG très précoces.

- L'héparine (de faible poids moléculaire), tout comme l'aspirine, agit sur les accidents thrombotiques. Deux injections sous cutanées par jour sont habituellement prescrites, notamment lorsqu'il existe de forts 
taux d'aPL et des antécédents d'accidents plus ou tardifs de la grossesse. Il n'est en général pas recommandé de l'associer aux corticoïdes étant donné les risques osseux identiques.

- Les gammaglobulines polyvalentes intraveineuses (IVIG) font partie de l'arsenal thérapeutique de nombreuses maladies autoimmunes [6]. Elles entraînent une immunosuppression et entrent en compétition avec les autoanticorps au niveau de leurs sites de fixation. Nous avons traité 62 femmes avec ISRG et aPL avec un taux de succès de $77 \%$ [8]. Un des avantages de ces IVIG est le peu de risques secondaires, mais le grand inconvénient est leur coût très élevé.

Chez les femmes traitées pour une insuffisance de l'immunité de tolérance par injection des lymphocytes du conjoint, une autoimmunité doit être recherchée, car, tout comme la grossesse, l'injection de lymphocytes peut faire apparaître des antiphospholipides dont il faudrait tenir compte lors de la grossesse à venir.

\section{AUTRES CAUSES IMMUNOLO- GIQUES POSSIBLES DES ISRG}

Dans l'avenir il est possible qu'un certain nombre de données nouvelles se confirment permettant de définir de nouveaux groupes d'ISRG immunologiques.

\section{Antigène HLA-G}

Le trophoblaste présente une résistance particulière aux cellules cytotoxiques et notamment celles qui sont présentes dans la décidua. Cette résistance est en partie due à l'absence des antigènes d'histocompatibilité et en particulier les antigènes HLA habituels (responsables de phénomènes de rejet de greffe). La résistance du trophoblaste est également imputable à la présence d'un antigène qui lui est propre, l'antigène HLA-G. Cet antigène d'empreinte génomique paternelle [4] ne s'exprime que sur cellules cytrophoblastiques de caractère invasif, en effet seules les cellules cytotrophoblastiques qui traversent le syncytium des villosités crampons, l'épithélium endométrial et sa membrane basale pour migrer dans l'endomètre et le premier tiers du muscle utérin, expriment l'antigène HLAG. Cet antigène confere aux cellules trophoblastiques une résistance particulière aux phénomènes de rejet. Ce fait est d'importance si l'on considère que la décidua est envahie de cellules lymphocytaires maternelles dont la capacité cytotoxique est reconnue bien que normalement moindre par rapport à celle des lymphocytes du sang périphérique.

La prolifération contrôlée des cellules trophoblastiques trouve ici, avec l'antigène HLA-G, très probablement un des facteurs de son équilibre. Dans certaines conditions cet équilibre pourrait être rompu. On connaît fort bien l'absence de rejet des môles hydatiformes où le phénomène d'empreinte génomique paternelle est particulièrement important. L'inverse doit très certainement exister et pourrait expliquer certains rejets de grossesse sans étiologie actuellement connue. On peut alors penser que l'expression de l'antigène HLA-G puisse être défectueuse ce qui entraînerait une invasion insuffisante des cellules trophoblastique dans la décidua, un retard du développement embryonnaire et la mort de l'embryon et par voie de conséquence ISG.

\section{II - Anticorps antitrophoblastes}

On peut aussi évoquer d'autres facteurs locaux, qui gêneraient l'invasion normale de cellules embryonnaires d'empreinte génomique paternelle normale. Ces facteurs pourraient être des anticorps, soit anticorps anti-HLA-G, soit d'autres anticorps comme les antitrophoblastes récemment décrits [5]. Les anticorps antitrophoblastes de spécificité non connue, ont été observés chez les femmes qui présentent des ISRG. Ces anticorps reconnaissent une glycoprotéine de $66,2 \mathrm{kD}$. Leur action pour- 
rait se faire par l'intermédiaire d'une inhibition de la fixation de l'urokinase plasmine activator (u-PA) sur leurs récepteurs au niveau du trophoblaste invasif. Ce mécanisme pourrait donc intervenir très précocément dès le début de la placentation. Les antitrophoblastes sont absents des sérums de femmes enceintes normales. Ils sont de nature IgG, IgM ou IgA. L'effet du traitement par corticoïdes, est d'autant plus efficace que le titre des anticorps est plus bas.

\section{Les embryotoxines}

Par ailleurs, il a été démontré que chez les femmes qui présentent des ISRG, les lymphocytes maternels stimulés par les antigènes paternels du trophoblaste peuvent produire une embryotoxine [5]. Elle peut être identifiée dans le sérum des femmes [2]. Ce facteur embryotoxique n'est pas observé chez les femmes qui mènent leurs grossesses normalement à terme [5].

\section{Déficience du système fibrinoly- tique}

Le dosage des inhibiteurs de l'activation du plaminogène pourrait également permettre de reconnaître la part de la mère de celle du père. Gris et al [9] ont récemment montré que les facteurs inhiteurs 1 et 2 de l'activation du plasminogen (PAI-1 et PAI-2) sont augmentés chez certaines femmes qui présentent des ISRG. L'identification d'une telle sous population d'ISRG pourrait conduire à un traitement préventif plus spécifique par héparine.

\section{CONCLUSION}

La participation du père est primordiale dans les mécanismes immunologiques $d u$ maintien de la greffe fœtale et dans leurs dysfonctionnement. Depuis une décennie une première étape consistait à rechercher un défaut de stimulation, par les antigènes paternels du fotus, des facteurs d'immunotolérance maternelle pour son fœus. L'injection de cellules du père a permis dans certains cas, de prévenir le rejet. Cette première étape consistait également à identifier les femmes, qui sous l'effet de l'intrusion des antigènes étrangers provenant $d u$ père, révélaient ou accentuait un état d'autoimmunité latent ou préexistant. L'utilisation de certains traitements préventifs, corticoïdes, aspirine ou gammaglobulines $\mathrm{IV}$, a pu permettre l'évolution normale d'un certain nombre nombre de grossesses vouées à l'échec.

Les recherches en cours permettront, sûrement dans un avenir proche, de confirmer certains mécanismes nouveaux des ISG et probablement de certaines stérilités inexpliquées. L'étude des antigènes fotaux d'empreinte génomique paternelle dans les différentes étapes de la placentation, seront probablement déterminants dans cette nouvelle étape.

\section{BIBLIOGRAPHIE}

1. DAYA S, GUNBY J, and The Recurrent Miscarriages Immunotherapy Trialists Group. The effectiveness of allogenieic leucocyte immunization in unexplained primary recurrent spontaneous abortion. Am J Reprod Immunol .1994; 32:294-302.

2. DOKRAS A, SARGENT IL, REDMAN CWG, BARLOW DG. Sera from women with unexplained infertility inhibit both mouse and human embryo growth in vitro. Fert Steril; 1993; 60: 285-92

3. GOSHEN R, BEN-RAFAEL Z, GOINK B, LUSTIG O, TANNOS V, DE GROOT N, HOCHBERG AA. - The role of genomic imprinting in implantation. Fert. Steril. 1994; 62: 903-10.

4. GRIS JC, NEVEU S, MARES P, BIRON C, HEDON B, SCHVED JF. - Plasma fibrinolytic activators and their inhibitors in women suffering from early recurrent abortion of unknown etiology. J Lab Clin Med, 1993; 122 : 606-15.

5. HILL JA, POLGAR K, BERNARD L, HARLOWL, ANDERSON DJ. - Evidence of embryo- and trophoblast-toxic cellular imm- 
nune reponses in women with recurrent spontaneous abortion. Am J Obstet Gynecol 1992; 166: 1044-52.

6. JUNGI TW, NYDEGGER UE.- Proposed mechanisms of action of intravenous IgG (IVIG) in autoimmune diseases. - Transf Sci. 1992; $13: 267-90$.

7. MCCRAE KR, DEMICHELE AM, PANDHI P, BALSAI MJ, SAMUELS P, GRAHAM CH, LALA PK, CINEZ DB. - Detection of antitrophoblast antibodies in sera of patients with anticardiolipine antibodies and fetal loss. Blood, 1993; 82: 2730-41.

8. REZNIKOFF-ETIEVANT MF, JARRAYA MA, DE LACHAUX V, MARPEAU L, PAPIERNICK E, NETTER A. Interruptions spontanées répétées de grossesse: aspects actuels des mécanismes immunologiques et traitements. Actualités Gynécologiques. 1991, 22: 159-171.
9. REZNIKOFF-ETIEVANT MF, DURIEUX I, HUCHET J, SALMON CH, NETTER A. Human MHC antigens and paternal leucocyte injections in recurrent spontaneous abortions. In: "Early pregnancy failure", RGOG eds. $1988: 375-84$.

10. The Recurrent Miscarriage Immunotherapy Trialists Group. World wide collaborative observational study and meta-analysis on allogeneic leucocyte immunotherapy for recurrent spontaneous abortion. Am J Reprod Immunol . 1994; 32, 55-77.

11. YOSHDA H, ITO K, KUSAKARI T, IDA K, IHARA Y, MORI T, MATSUMURA M. Removal of maternal antibodies from women with repeated fetal loss due to $\mathrm{P}$ blood incompatibility. Tranfusion 1994; 34: 702-5. 